ARTICLE

DOI: $10.1038 /$ ncomms10546

OPEN

\title{
Toughness and strength of nanocrystalline graphene
}

\author{
Ashivni Shekhawat ${ }^{1,2,3} \&$ Robert O. Ritchie ${ }^{1,2}$
}

Pristine monocrystalline graphene is claimed to be the strongest material known with remarkable mechanical and electrical properties. However, graphene made with scalable fabrication techniques is polycrystalline and contains inherent nanoscale line and point defects-grain boundaries and grain-boundary triple junctions-that lead to significant statistical fluctuations in toughness and strength. These fluctuations become particularly pronounced for nanocrystalline graphene where the density of defects is high. Here we use large-scale simulation and continuum modelling to show that the statistical variation in toughness and strength can be understood with 'weakest-link' statistics. We develop the first statistical theory of toughness in polycrystalline graphene, and elucidate the nanoscale origins of the grain-size dependence of its strength and toughness. Our results should lead to more reliable graphene device design, and provide a framework to interpret experimental results in a broad class of two-dimensional materials.

\footnotetext{
${ }^{1}$ Materials Sciences Division, Lawrence Berkeley National Laboratory, Berkeley, California 94720, USA. ${ }^{2}$ Department of Materials Science \& Engineering, University of California, 324 Hearst Memorial Mining Building, MC 1760, Berkeley, California 94720, USA. ${ }^{3}$ Miller Institute for Basic Research in Science, Berkeley, California 94720, USA. Correspondence and requests for materials should be addressed to A.S. (email: shekhawat.ashivni@gmail.com) or to R.O.R. (email: roritchie@lbl.gov).
} 
T he high strength of graphene combined with its exceptional electronic $^{1,2}$, optical ${ }^{3}$ and thermal properties ${ }^{4}$ has made it an ideal material for many fascinating applications, including flexible electronic displays ${ }^{5}$, corrosion-resistant coatings $^{6}$, biological devices ${ }^{7,8}$ and many more ${ }^{9}$. While each of these applications exploits a different key property of graphene, they all implicitly depend on its exceptional mechanical properties for structural reliability ${ }^{10}$. However, such mechanical reliability of graphene is impacted by atomic defects in its structure. While the effect of relatively simple defects, such as isolated dislocation cores or a few special grain boundaries (GB), on the strength of graphene is understood ${ }^{11-17}$, the statistical fluctuations in strength and toughness due to the randomness in polycrystalline nanostructure remains largely unexplored.

Strength and toughness are arguably the two most important properties of a structural material. While strength is generally a function of the material's resistance to deformation, toughness represents its resistance to fracture. In most materials, these properties tend to be mutually exclusive ${ }^{18}$. There are conflicting experimental reports whether the strength of polycrystalline graphene is actually a function of grain size ${ }^{19-21}$ making the role of simulation and theory more critical. Understanding these statistical fluctuations has become important in light of the fact that graphene synthesized with chemical vapour deposition (CVD) is polycrystalline, and this method is being used to manufacture more than $300,000 \mathrm{~m}^{2}$ of graphene annually ${ }^{22,23}$.

It is well-established that the strength and toughness of polycrystalline solids is strongly influenced by their granular structure. For instance, nanocrystalline metals are invariably significantly harder and much less ductile than their microcrystalline counterparts. This is due to the well-known 'Hall-Petch effect', wherein the motion of dislocations, and thereby plastic flow, is impeded by the presence of $\mathrm{GB}^{24}$. On the other hand, dislocations are typically not mobile in brittle materials, such as ceramics (and graphene), and thus the Hall-Petch effect is not observed in these materials. An entirely different mechanism leads to grain-size-dependent strength in brittle ceramics where the length of the typical extrinsic crack-like flaw (inclusion/ porosity) relative to the grain size determines the characteristic strength $^{25,26}$. In contrast to typical bulk brittle materials such as ceramics, graphene can be fabricated in a much cleaner and controlled environment, thus making the presence of large extrinsic defects unlikely ${ }^{22}$. In the absence of such extrinsic defects, the fluctuations in strength must arise from intrinsic atomic defects inherent to the granular nanostructure. The traditional theories developed for brittle ceramics with large extrinsic flaws are thus not applicable for strength fluctuations due to these intrinsic defects. In graphene these defects are GBs and triple junctions (TJs). Although a GB is an interface between crystalline regions of different lattice orientations and a TJ is the intersection of three such interfaces, in graphene GBs and TJs are typically composed of pentagon-heptagon defects, also known as five to seven defects (Fig. 1) 2 $^{2-30}$. These defects involve significant residual stresses and act like stress concentrators.

In this work, we develop an understanding of the grain-sizedependent statistical fluctuations in strength and toughness of polycrystalline graphene by using a combination of weakest-link statistics, continuum elastic theory and large-scale molecular dynamics (MD) simulations. Here we find that while the strength of a brittle polycrystalline graphene sheet is dictated by the weakest flaw in the entire sheet, its toughness is conversely influenced by the GB nearest to the crack tip. We develop theories to capture these two markedly different mechanisms. We believe that the theoretical framework developed here will be applicable to a large class of emerging two-dimensional (2D) materials.
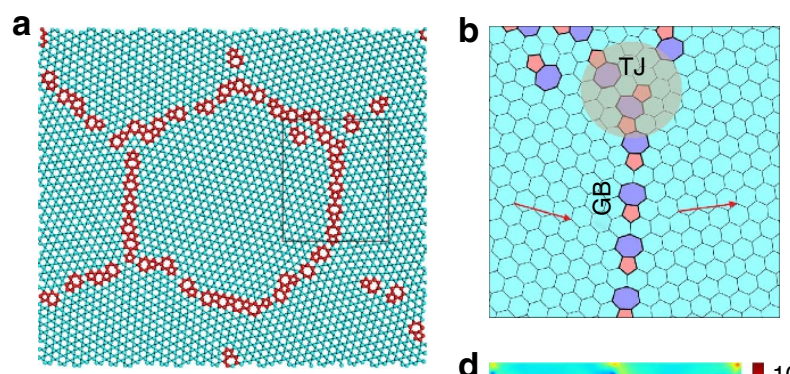

c
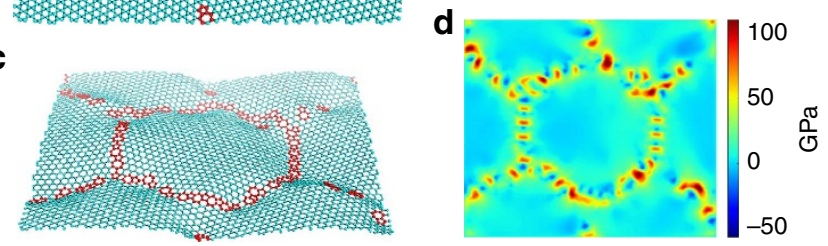

Figure 1 | Structure of polycrystalline graphene. (a) A one-atom-thick polycrystalline graphene sheet composed of carbon atoms arranged in regular hexagonal rings, except at the grain boundaries (GBs) and triple junctions (TJs). The defected atoms at the GBs and TJs that are part of nonhexagon rings are drawn in red for clear identification. (b) A zoom-in of the area marked in $\mathbf{a}$. The red arrows indicate the orientation of the grains on either side of the GB; the GB itself is composed of rings of five (coloured pink) and seven (coloured blue) carbon atoms. These are the dislocation cores with the shortest Burgers vectors in graphene, and thus represent low-energy configurations of GBs. A TJ formed at the intersection of three GBs is highlighted. (c) A perspective view of the graphene sheet showing its 3D structure. (d) The principal residual stresses in the graphene sheet are in units of GPa. The grain interiors are defect free, while the GBs and TJs have significant residual stresses, and thus are the weak points where fracture nucleates.

\section{Results}

Statistical distribution of strength. We use MD simulations to gain insights into the statistical distribution of polycrystalline strengths ${ }^{31-33}$. We simulate fracture in over 19,000 polycrystalline graphene sheets with random grain shapes and orientations at several different combinations of sheet size, grain size and strain rate. The details of our simulations can be found in the Methods section and in Supplementary Note 1. Figure 2a shows a schematic representation of one such simulation. Figure $2 b, c$ shows the snapshots of a polycrystalline simulation at zero stress, and at peak stress just before global failure, respectively. Residual stress at $\mathrm{GB}$ and $\mathrm{TJ}$ defects can be seen in Fig. 2b; the length-scale associated with these stresses is sub-nanometer, showing that the fluctuations are truly atomicscale phenomena. Figure $2 c$ shows that fracture originates at a grain boundary and then progresses through rest of the polycrystal. This observation is generic and fracture always originates at a GB or TJ in the several thousand polycrystals that we have simulated. This is not surprising since the interiors of the grains are defect-free and have no residual stresses. Once fracture originates, the incipient crack can extend in an intragranular or intergranular manner depending on the details of the grain orientation and loading direction.

Traditionally, the statistical distribution of fracture strengths in brittle materials is understood in terms of Weibull theory ${ }^{34-37}$. According to Weibull, the survival probability of brittle materials, defined as the probability that a volume $V$ of the material survives at a stress $\sigma$, is of the form $S(\sigma)=\exp \left(-V\left(\frac{\sigma-\sigma_{0}^{\prime}}{v^{\prime}}\right)^{m}\right)$ where the lower-bound strength, $\sigma_{0}^{\prime}$, and $v^{\prime}$ are, respectively, the location and scale parameters, and $m$ is the Weibull modulus. These parameters are generally evaluated by data fitting; however, in our case, fitting this from simulation data for each combination of grain size, strain rate and system size would give a different value 
a

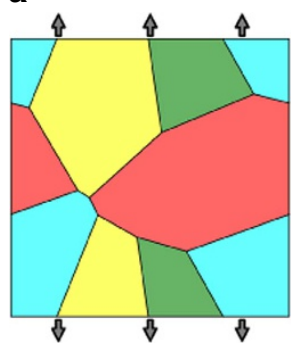

b

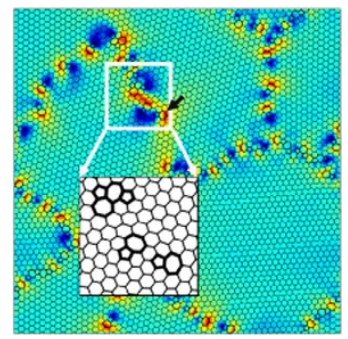

c

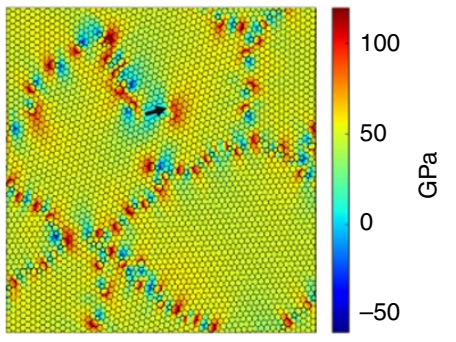

Figure 2 | Simulation of damage and failure during tensile loading of polycrystalline graphene. (a) A schematic representation of the fracture simulation of a 2D periodic graphene nanoscale polycrystal. Each colour represents a grain within which the lattice orientation is fixed. The polycrystal is loaded at a constant strain rate. (b) Residual stress in a polycrystal; the principal stress is plotted in units of GPa. The interiors of the grains are stress free while there is significant residual stress at the GBs and TJs. The black arrow points to the large stress concentration at the tail of the pentagon-heptagon defect where fracture ultimately nucleates. The inset shows a zoom-in of the atomic configuration. (c) Principal stress just before global fracture. The crack nucleates at the GB defect and extends through the adjacent crystal. Notice that there are a few other incipient cracks that do not go unstable.

for these parameters, and thus would not provide any physical insight into the dependence of these parameters on the nanostructural features such as grain size. As our goal is to gain such insight, we combine Kramer's rate theory with extreme value statistics to derive an expression for the survival probability that explicitly accounts for the dependence on the physical atomistic and structural variables of interest. Similar expressions have been derived in various contexts previously, dating back at least to the work of Tobolsky and Eyring ${ }^{38}$, among other more recent studies ${ }^{39-43}$

The specific details of our derivation can be found in the Supplementary Note 2; we present here the main result. We consider a polycrystalline graphene sheet of linear size $L$ with a linear grain size $\mu$. The sheet is loaded uniaxially at a constant strain rate $\dot{\epsilon}$ at a fixed temperature of $300 \mathrm{~K}$. Thus, the stress at time $t$ is given by $\sigma(t)=\left(\frac{Y}{1-\eta^{2}}\right) \dot{\epsilon} t$, where $Y$ is the Young's modulus and $\eta$ is the Poisson's ratio. Our main result for polycrystalline strength gives the following expression for the survival probability of the sheet loaded at strain rate $\dot{\epsilon}$ up to a stress $\sigma$ :

$$
S(\sigma \mid L, \mu, \dot{\epsilon})=e^{-\frac{L^{2} i_{0}}{\mu^{2} \dot{\sigma}}\left(\frac{\sigma-\sigma_{0}}{v}\right)^{m}}
$$

where $\dot{\epsilon}_{0}$ is a reference strain rate for normalization, and $\sigma_{0}, v$ are the rescaled location and scale parameters. As opposed to the usual Weibull form, in equation (1) the effect of structural parameters such as system size, grain size and loading rate is captured by a single non-dimensional parameter $k \equiv L^{2} \dot{\epsilon}_{0} / \mu^{2} \dot{\epsilon}$, while the rescaled parameters $\sigma_{0}, v, m$ are not affected by these details and are true material properties. We arrive at this particularly simple result by assuming that the individual defects in the graphene sheet are non-interacting, and that the loading is slow enough so that a thermal quasi-equilibrium is achieved. The dependence on $L^{2} / \mu^{2}$ is due to the fact that fracture initiates at GB and TJ defects, and the number of such defects in a graphene sheet scales as $\sim L^{2} / \mu^{2}$. The factor $\dot{\epsilon}_{0} / \dot{\epsilon}$ is due to the fact that a lower strain rate gives more opportunity for thermal nucleation of fracture at the defects. It is interesting to note that similar ideas are applicable to dislocation nucleation in plasticity ${ }^{41-46}$, where it has been noted that the likelihood of survival of a metallic nanopillar under stress decreases with time due to nucleation events. Our model is a 'weakest-link' model, since we assume that the graphene sheet fractures as soon as its weakest GB/TJ defect becomes unstable. One particularly interesting outcome of our analysis is that the strength of graphene is a function of the ratio $L^{2} / \mu^{2}$; thus there is no one well-defined value of strength at a given grain size. As we shall show, this behaviour is in contrast to the toughness, which is well-defined for a given grain size. Finally, it is worth noting that in our derivation, the connection between nucleation theory and Weibull analysis is made via the mathematical theory of extreme value statistics (as shown in Supplementary Equation 8$)^{47,48}$.

We test our theoretical result with large-scale MD simulations. We perform extensive statistical sampling for 24 different combinations of the parameters $L, \mu, \dot{\epsilon}$. Figure 3 a shows the variation of the survival probability with grain size, while Fig. $3 \mathrm{~b}$ shows the effect of strain rate. A joint fit of the survival probability for the entire data set using the form given in equation (1) is obtained from the maximum likelihood estimator (see Supplementary Note 3 for details). This fit yields a Weibull modulus of $m=10.7$, a location parameter of $\sigma_{0}=19.5 \mathrm{GPa}$ and a scale parameter of $v=53.2 \mathrm{GPa}$. Note that the Weibull modulus and the scale parameter are a measure of the statistical variability of the strength, and the s.d. of the strength distribution scales as $\sim(v / m) k^{-1 / m}$. A modulus of $\sim 10$ implies a moderate amount of variability from a structural engineering viewpoint. By comparison, the Weibull modulus for brittle glasses is $\sim 1-3$, while that for well-designed ceramics is $\sim 10-20$. The Weibull modulus has been interpreted in a similar manner in nanoscale plasticity, where it is considered to be a general measure of strength fluctuations ${ }^{44,46}$. The location parameter simply indicates a lower bound strength. Figure $3 \mathrm{c}$ shows that with the values of these parameters for graphene, all the data for the various combinations of $L, \mu, \dot{\epsilon}$ collapses on single line, validating our theoretical result. Equation (1) further predicts that the mean fracture strength of graphene should scale as:

$$
\sigma=\sigma_{0}+v\left(\frac{\dot{\epsilon}}{\dot{\epsilon}_{0}}\right)^{\frac{1}{m}}\left(\frac{\mu}{L}\right)^{\frac{2}{m}} \Gamma\left(1+\frac{1}{m}\right)
$$

where $\Gamma(\cdot)$ is the Gamma function. Figure $3 \mathrm{~d}$ presents a validation of this relation. Note that after $\sigma_{0}, v, m$ have been obtained by fitting the survival probability to equation (1), there are no more free parameters, and thus the graph in Fig. $3 \mathrm{~d}$ contains no free parameters. The scaling of the mean strength with grain size in equation (2) is similar to the result reported by Sha et al. ${ }^{49}$; however, they did not model the system size and strain-rate dependence.

Statistical distribution of toughness. The strength of a material quantifies its failure in response to a state of large homogeneous stress, although in practice the state of stress is rarely homogeneous; rather, some regions of the material experience much higher stress than others. This is often due to the presence of stress singularities associated with sharp cracks and corners. The fracture toughness quantifies such failure in response to the 

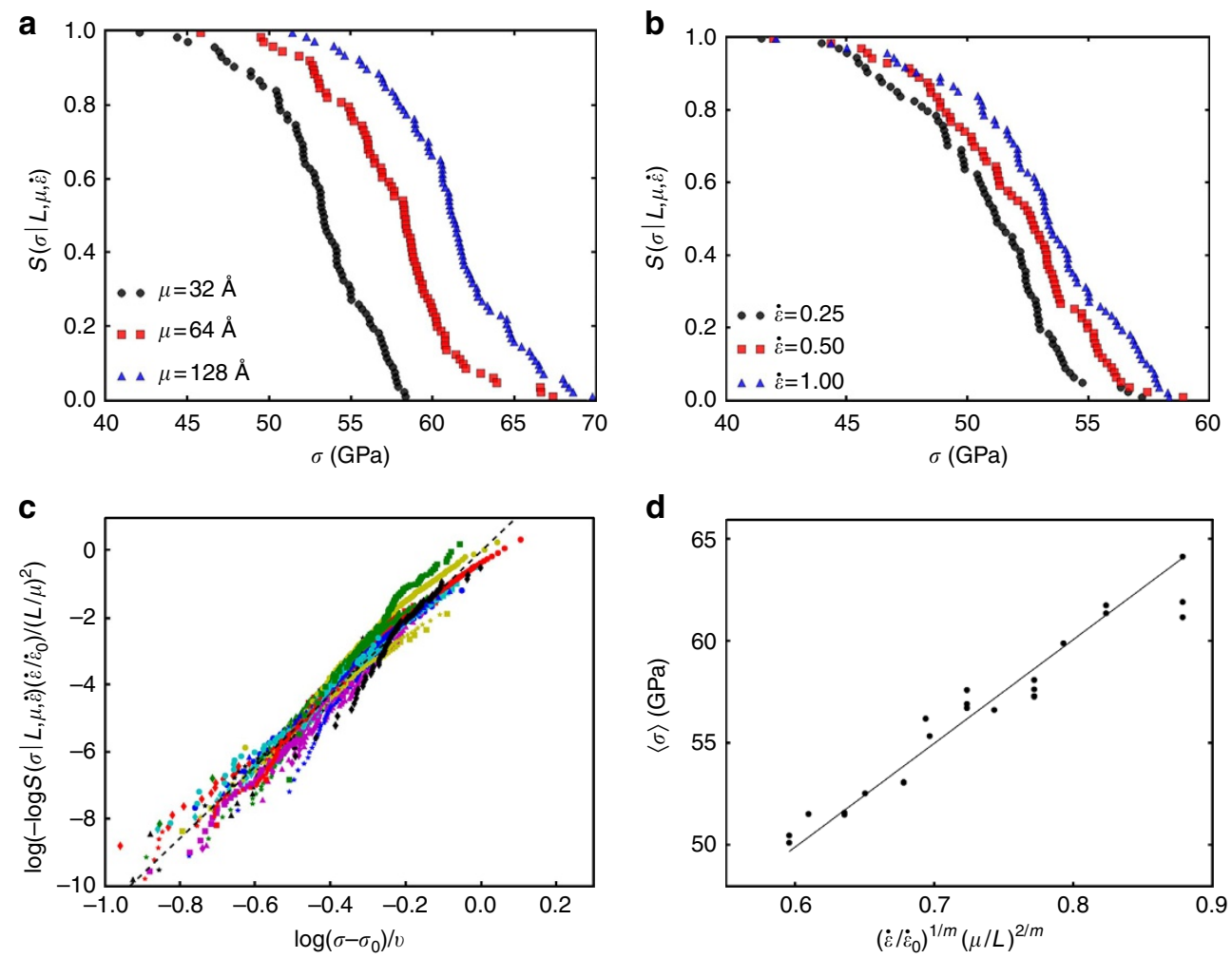

Figure 3 | Statistical distribution of strengths in polycrystalline graphene. (a) The variation of the survival probability in nanocrystalline graphene with grain size $\mu$ at $L=256 \mathrm{~A}, \dot{\epsilon}=10^{9} \mathrm{~s}^{-1}$. At fixed stress, the smaller grain size has lower survival probability due to a higher density of GB and TJ defects. (b) The variation of survival probability with strain rate $\dot{\epsilon}$ (in units of $10^{9} \mathrm{~s}^{-1}$ ) at $L=256 \AA, \mu=32 \AA$. (c) A data collapse of the survival probability (obtained from MD simulations) according to equation (1) for 24 different combinations of $L, \mu, \dot{\epsilon}$ (see Methods section). The dashed black line is a guide to the eye and shows the prediction of equation (1). The simulation data closely follows the predicted form. (d) A collapse of the mean failure stress as predicted by equation (2). Note that this collapse has no free parameters.

stress singularity due to the presence of a crack. Not surprisingly, the toughness is one of the most important mechanical properties of a material. For nominally brittle materials, the fracture toughness can be evaluated in terms of the critical stress-intensity factor, $K_{\mathrm{I}_{\mathrm{cr}}}$, which is a measure of the strength of the stress singularity at the crack tip (the stress intensity specifically quantifies the magnitude of the elastic stress and displacement fields at the crack tip). Griffith's criterion establishes a lower bound for the critical stress-intensity factor as $K_{\mathrm{I}_{\mathrm{cr}}} \geq \sqrt{2 \gamma Y}$, where $\gamma$ is the energy required to create the fresh crack surface. However, the Griffith theory does not account for statistical fluctuations in $K_{\mathrm{I}_{\mathrm{cr}}}$ due to variations in the local nanostructure of GBs and TJs. Here we develop a theory to account for these factors.

We use MD simulations to explore the statistical fluctuations in fracture toughness and its dependence on grain size in graphene. Figure 4a shows a schematic representation of our simulations. We simulate the initial advance of semi-infinite cracks in graphene polycrystals by imposing suitable fixed boundary conditions away from the crack tip, and evolving the atoms near the crack tip with canonical NVT dynamics; specific details can be found in the Methods section. As shown in Fig. 4b, the stress singularity at the crack tip interacts with the GB and TJ defects in the immediate vicinity of the tip. The critical stress-intensity factor needed to initiate crack advance depends on the local nanostructure; as an arbitrary crack would experience a random sampling of this nanostructure, there is significant statistical spread in the values of $K_{\mathrm{I}_{\mathrm{cr}}}$ and thus it is meaningful to define a grain-size-dependent survival probability, $S\left(K_{\mathrm{I}} \mid \mu\right)$, as the probability that a polycrystal with grain size $\mu$ survives an applied stress-intensity factor $K_{\mathrm{I}}$. Figure $5 \mathrm{~b}$ shows the numerically obtained survival probability from our $\mathrm{MD}$ analysis on polycrystals with different grain sizes. This figure clearly shows that although there is significant spread in the observations, there is a strong dependence on grain size.

In general, we have observed that cracking progresses by breaking the bonds associated with the pentagon-heptagon defects near the crack tip. In fact, due to lack of plasticity, the stress concentration at the crack tip in graphene is so strong that the first bond to break is almost always within $10 \AA$ of the crack tip. Because the polycrystalline morphology is generated randomly, as the grain size is increased it becomes more likely that there are no defects near the crack tip. If there are no GBs or TJs near the tip, then the toughness is simply given by the toughness of the monocrystal that contains the crack tip. Since the monocrystal will have an arbitrary orientation, a part of the statistical spread in our simulation results is due to the variation in fracture toughness of pristine graphene with orientation. For the AIREBO empirical potential ${ }^{50,51}$ used in our MD simulations, we find that the elastic modulus $Y=858 \mathrm{GPa}$, and $\gamma$ varies between 5.9 and $6.3 \mathrm{~J} \mathrm{~m}^{-2}$ for single crystals depending on the orientation, resulting in an orientation-dependent Griffith estimate of fracture toughness between 3.2 and $3.3 \mathrm{MPa} \mathrm{m}^{1 / 2}$ for monocrystalline graphene. In practice the observed values of the toughness of brittle solids are slightly higher than the Griffith bound due to such effects as lattice trapping and crack roughness. We simulate crack advance in graphene monocrystals with random orientations and find that the resulting distribution of 
a

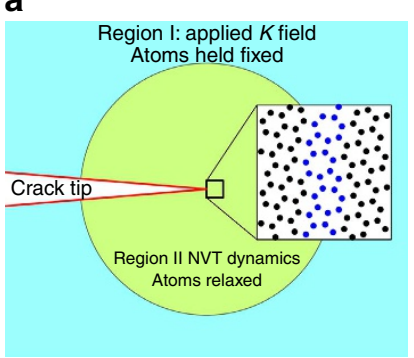

b

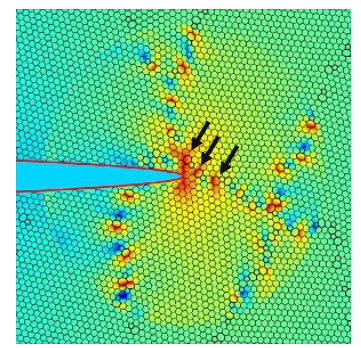

c

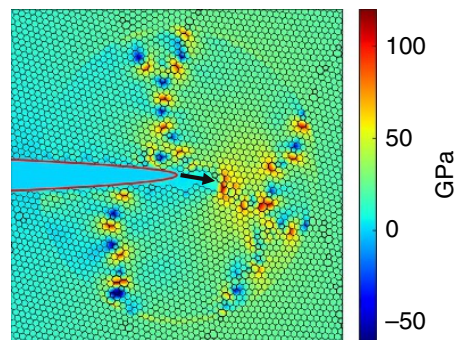

Figure 4 | Fracture simulations in polycrystalline graphene. (a) A schematic representation of the simulation of initial crack advance in nanocrystalline graphene. A prescribed $K_{1}$-field is applied by holding the atoms outside the circular region of radius $100 \AA$ fixed at the positions given by the LEFM solution. The atoms inside the circular region are allowed to relax with NVT dynamics. Initial crack advance occurs when the stress intensity $K_{1}$ is sufficiently large. (b) The stress field in a polycrystal where the atoms outside a $100 \AA$ radius from the crack tip are held fixed at the LEFM displacement field for $K_{1}=4 \mathrm{MPa} \cdot \mathrm{m}^{1 / 2}$. The black arrows show the enhancement of the crack-tip stress concentration by the GB defects. (c) The crack extends along the GB as indicated by the black arrow. The stress concentration is relieved after crack extension.

$S_{\mathrm{cr}}\left(K_{\mathrm{I}}\right)$ is well-described by a Gaussian distribution with mean 3.9 $\mathrm{MPa} \cdot \mathrm{m}^{1 / 2}$ and s.d. $0.4 \mathrm{MPa} \cdot \mathrm{m}^{1 / 2}$. This somewhat accounts for the behaviour in the upper tail (large $K_{\mathrm{I}}$ ) of Fig. 5b, but the interesting grain-size-dependent behaviour for smaller values of $K_{\mathrm{I}}$ needs to be explored further. We note that the trend of increasing toughness with grain size is opposite to the trend reported elsewhere ${ }^{52}$. Presumably, this is due to the fact that we are measuring the value of $K_{\mathrm{I}_{\mathrm{cr}}}$ strictly as a crack-initiation toughness (at instability) while ref. 52 reports a propagation toughness; moreover, their simulations seem to display crack bridging with single atom carbon chains (a non-physical phenomenon typical of the AIREBO potential, which we avoid in this work by the design of our simulations).

We now develop a theory to explain the grain-size dependence of $S\left(K_{\mathrm{I}} \mid \mu\right)$ in graphene. To maintain theoretical tractability, we do not account for loading-rate dependence, and all our simulations of initial crack advance are performed in the quasistatic limit. The stress field in the region of $K$-dominance is known from linear-elastic fracture mechanics (LEFM). For ductile materials with brittle inclusions, the statistical variation in toughness can be obtained by integrating the Weibull-type expression of stress survival probability equation (1) over the LEFM crack-tip stress field ${ }^{33,54}$. A similar method has been used to study the nucleation of dislocations under an indenter tip in plasticity ${ }^{44,46}$. However, this technique implicitly assumes that a large population of defects is sampled by each crack tip (or indenter tip in plasticity); while this assumption is valid for ductile materials with large crack-tip process zones, it is clearly violated for brittle materials like graphene where the crack tip samples only a few defects within a few angstroms of the tip. Thus, a new approach is needed. It can be argued that the effect of TJs on the distribution of $K_{\mathrm{I}}$ should be minimal, since the probability of finding a TJ near a crack tip is much smaller than the probability of finding a GB nearby. Figure $5 \mathrm{a}$ shows a schematic representation of our model. We consider an arbitrary GB a distance $l$ ahead of the crack tip, inclined at an angle $\phi$ to the crack. Each point on this GB experiences a different normal stress $\sigma_{\mathrm{n}}$ that can be calculated from LEFM to be $\sigma_{n}=\frac{K_{\mathrm{I}}}{\sqrt{2 \pi r}} \cos \left(\frac{\theta}{2}\right)\left(1+\sin \left(\frac{\theta}{2}\right) \sin \left(\frac{3 \theta}{2}-2 \phi\right)\right)$. We define $r^{*}, \theta^{*}$ as the point at which the GB experiences the maximum normal stress, $\sigma_{\mathrm{n}}^{*}$. Note that $\sigma_{\mathrm{n}}^{*}$ is implicitly a function of $l$ since $r^{*}=\frac{l \sin \phi}{\sin \left(\phi-\theta^{*}\right)}$. We make the assumption that this GB survives at stress intensity $K_{\mathrm{I}}$ if it can survive the normal stress $\sigma_{\mathrm{n}}^{*}$. We measure the GB survival probability, $S_{\mathrm{GB}}\left(\sigma_{\mathrm{n}}\right)$, for applied normal stress by simulating over 4,000 GBs (see Methods, Supplementary Note 1 and Supplementary Fig. 1); the resulting survival probability and probability density are shown in Fig. $5 c$. The polycrystalline survival probability can then be written as:

$S\left(K_{\mathrm{I}} \mid \mu\right)=\int_{l_{\mathrm{c}}}^{\infty} \int_{0}^{\pi} S_{\mathrm{GB}}\left(\sigma_{\mathrm{n}}^{*}\left(\phi, l, K_{\mathrm{I}}\right)\right) p(\phi) \mathrm{d} \phi p(l \mid \mu) \mathrm{d} l * S_{\mathrm{cr}}\left(K_{\mathrm{I}}\right)$,

where $p(\phi)$ is the probability density of the random GB orientation angle $\phi, p(l \mid \mu)$ is the grain-size-dependent probability density of the GB distance from crack tip, and $l_{c}$ is a lower cutoff due to the discreteness of the lattice. Equation (3) essentially means that the polycrystal survives a stress intensity $K_{\mathrm{I}}$ if the nearest GB ahead of the crack tip and the crystal containing the crack survive. Since the polycrystal has random boundary orientations, the distribution of $\phi$ is uniform, giving $p(\phi)=1 / \pi$. The distribution of the GB distance from the crack tip is measured from the randomly generated polycrystals to be a half-Gaussian distribution, $\quad p(l \mid \mu)=\frac{2 e^{-\left(\frac{l^{2}}{2 \alpha^{2} \mu^{2}}\right)}}{\sqrt{2 \pi \alpha^{2} \mu^{2}}}$, where the parameter $\alpha$ is equal to 0.64 . A collapse of the probability density of the measured distance to the nearest GB ahead of the crack tip according to this form is shown in Fig. 5d. Finally, we take $l_{\mathrm{c}}$ to be equal to the distance between the centres of the next nearest hexagons in graphene $(=3 a$, where $a$ is the carboncarbon bond length in graphene). Note that there are no free parameters in equation (3). However, since it is unreasonable to assume that only the nearest GB directly ahead of the crack tip contributes to fracture, we leave $\alpha$ as a free parameter when fitting equation (3) to numerical data. The solid lines in Fig. 5b show a comparison of the predictions of the grain-size-dependent polycrystalline fracture toughness obtained from equation (3) with the numerical data obtained from extensive simulation. It should be noted that $\alpha=0.7$ is obtained from the fitting process, and is very close to the measured value of 0.64 . Thus, we are able to derive a formula for the statistical fluctuations in polycrystalline toughness that has only one free parameter, the value of which can also be measured to good accuracy from the polycrystal geometry.

\section{Discussion}

Our theoretical results offer important insights into the grain size and sample-size dependence of strength and toughness of graphene. The scaling of strength with grain size in traditional metals is understood in terms of the Hall-Petch effect ${ }^{24}$, while the analogous results in ceramics are explained with the inverse square-root scaling ${ }^{25}$. The current paper provides the corresponding scaling relations for the strength of graphene and other brittle 2D materials equations (1) and (2), while equation (3) provides the scaling relation for the toughness of $2 \mathrm{D}$ 
a
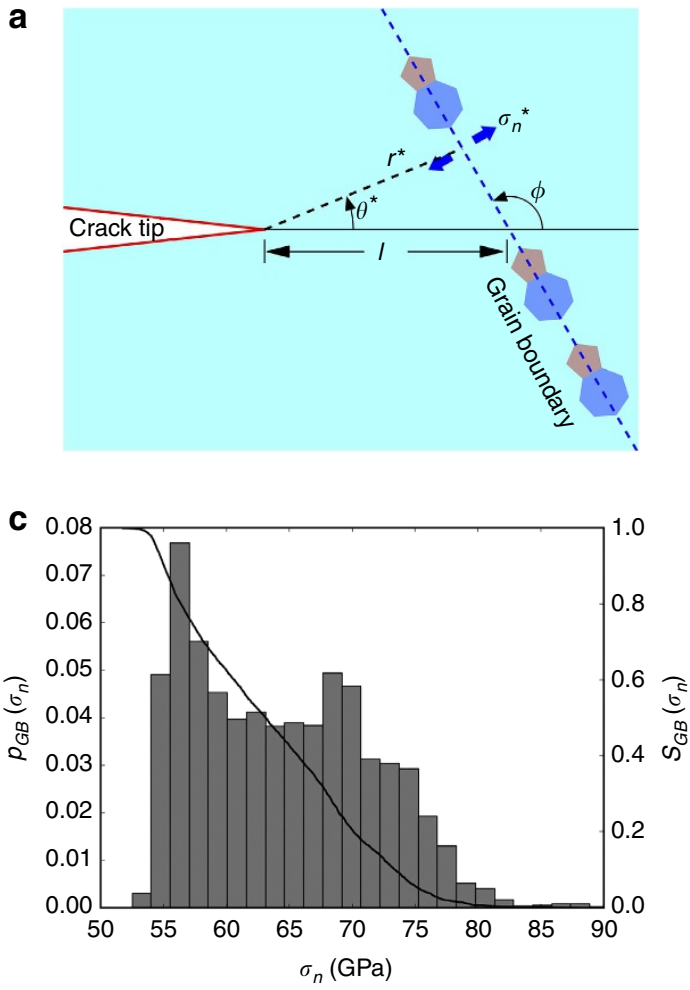
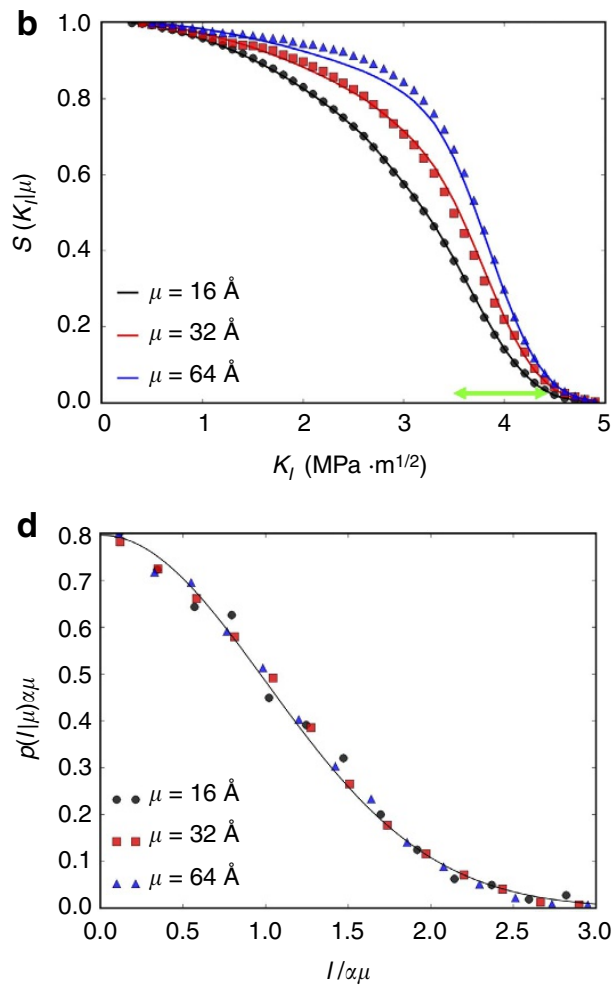

Figure 5 | Statistical distributions of toughness in polycrystalline graphene. (a) A schematic representation of our model for the toughness of nanocrystalline graphene. A GB a distance / ahead of the crack tip experiences the maximum normal stress at the point $\left(r^{\star}, \theta^{\star}\right)$. We assume that the polycrystal survives if the nearest GB and the crystal containing the crack survive the stress due to the crack. (b) A comparison of the numerically measured (from MD) survival probability for various grain sizes $\mu$ with the predictions of equation (3). The theoretical predictions are shown in the solid lines, while the MD data are shown in the symbols. Agreement between theory and simulation is evident. The toughness range for monocrystalline samples is indicated by the green line (the spread is due to change in crack orientation with respect to crystal axis). It is evident that crack-trapping due to GBs is minimal, and in most cases GBs lower toughness by facilitating crack advance. (c) The grain-boundary survival probability and failure probability density under applied normal stress measured from simulation of over 4,000 GBs. (d) The distribution of the distance of the nearest GB ahead of the crack tip measured from the randomly generated polycrystals with different grain sizes. The solid line is a plot of the half-Gaussian fit.

materials. As such, these relationships can serve a role analogous to the Hall-Petch effect in design of 2D materials. An understanding of the scaling relations is needed to design any device where the structural integrity of a graphene component is to be ensured. Their role is actually more significant in $2 \mathrm{D}$ materials because it is difficult to conduct systematic experimental investigations of their mechanical properties. The fact that the strength of polycrystalline graphene has never been measured under uniaxial tension, and that its toughness has been measured only once, is a true testament to this experimental challenge.

Our prediction of a mean toughness of $3-4 \mathrm{MPa} \cdot \mathrm{m}^{1 / 2}$ compares favourably with the only reported experimental measurement of the toughness of graphene ${ }^{10}$. Note that this toughness is not high; specifically, it is three to four times tougher than silicon and pyrolytic carbon ${ }^{55}$ yet $\sim 20$ to $50 \%$ less tough than polycrystalline diamond ${ }^{56}$. We have found that the distribution of toughness, as well as strength, in polycrystalline graphene is strongly dependent on the grain size. However, at larger grain sizes the distribution of toughness becomes less sensitive to grain size. We predict that for grain sizes larger than $256 \AA$, the toughness will be essentially independent of the grain size. Thus the toughness dependence of grain size is a phenomena limited to nanocrystalline graphene. In contrast, the strength will continue to be grain-size and sample-size dependent, and the experimental results will have to be scaled with the Weibull from equation (1) to get true material properties. We predict a Weibull modulus of $\sim 10$ for polycrystalline graphene. We note that even though our simulations are for nanocrystalline grains, we expect our results to be valid for much larger micrometer-sized grains, since no new physics is expected to emerge at the intermediate length scales. Our results are consistent with the observations that presence of GB reduces the strength of polycrystalline graphene ${ }^{20,21}$. Finally, we note that our simulations consider only the equilibrium geometrically necessary dislocations, and ignore the nonequilibrium defects such as disclinations ${ }^{57,58}$. It has been demonstrated that these latter defects can have a significant effect on the strength of graphene ${ }^{57,58}$. Our theory can be modified to include the effect of such defects if the statistical information regarding them, such as their density, can be obtained experimentally.

What do these results mean in practical terms? The first measurement of the strength of pristine monocrystalline graphene reported an intrinsic strength of about $130 \mathrm{GPa}$, and a Young's modulus of about $1 \mathrm{TPa}$. In practical terms these results mean that a soccer ball can be placed on a single sheet of graphene without breaking it. What object can be supported by a corresponding sheet of polycrystalline graphene? It turns out that a soccer ball is much too heavy, and polycrystalline graphene can only support a ping-pong ball. Still remarkable for a one-atomthick material, but not quite as breathtaking anymore.

\section{Methods}

Details of simulations. All numerical simulations were performed with the LAMMPS $^{59}$ code by using the AIREBO ${ }^{50,51}$ empirical interatomic potential, with the interaction cutoff parameter set to $1.92 \AA$ (ref. 11). The simulations were 
conducted in the NVT ensemble (the canonical ensemble with constant number of particles, volume and temperature) at temperature $T=300 \mathrm{~K}$. The random grain morphology was generated by randomly choosing the required number of grain 'centres' and generating the boundaries of the granular domains with a Voronoi construction. The crystalline orientations within each grain were also taken to be random. The atomic positions, particularly at the GB, were generated by a recently proposed algorithm that yields well-annealed $\mathrm{GBs}^{60}$. Even though this algorithm was proposed for generation of GBs, it can be used successfully to generate wellannealed polycrystalline samples (see Supplementary Note 1 and Supplementary Figs 2 and 3). All structures used in our strength and toughness simulations were prepared running NVT dynamics at $T=300 \mathrm{~K}$ for $1 \mathrm{ps}$, followed by energy minimization using the conjugate gradient algorithm (allowing out of plane deformations, and allowing the simulation cell dimensions to change to attain zero stress).

Simulation of strength. For the simulations of polycrystalline strength, a constant strain rate $\dot{\epsilon}$ was imposed using the SLLOD equations ${ }^{61}$ as implemented in LAMMPS, and the stress response was measured; the largest observed stress was taken to be the ultimate strength of the sample. We performed simulations for the following 24 combinations of the parameters $L, \mu, \dot{\epsilon}:(64,32,1),(128,64,1)$,

$(128,32 \sqrt{2}, 1),(128,64 / \sqrt{3}, 1),(128,32,1),(128,32 \sqrt{2}, 0.5),(128,64 / \sqrt{3}, 0.5)$, $(128,32 \sqrt{2}, 0.25),(128,64 / \sqrt{3}, 0.25),(256,128,1),(256,64 \sqrt{2}, 1),(256,64,1)$,

$(256,32 \sqrt{2}, 1),(256,128 \sqrt{2} / 5,1),(256,32,1),(256,64 \sqrt{2}, 0.5),(256,32 \sqrt{2}, 0.5)$, $(256,128 \sqrt{2} / 5,0.5),(256,32,0.5),(256,64 \sqrt{2}, 0.25),(256,32 \sqrt{2}, 0.25)$, $(256,128 \sqrt{2} / 5,0.25),(256,32,0.25),(512,32,1)$, where the units of $L, \mu$ are $\AA$, and those of $\dot{\epsilon}$ are $10^{9} \mathrm{~s}^{-1}$. The number of simulations performed for statistical sampling was $10^{4}, 10^{3}, 10^{2}$ for $L=64,128,256,512 \AA$, respectively. See Supplementary Figs 4 and 5 for the stress-strain response and snapshots of a representative simulation.

Simulation of toughness. For polycrystalline toughness, we simulated systems of length $L=256 \AA$ with a crack tip placed at the centre of the simulation box. The atoms outside a radius of $100 \AA$ from the crack tip were fixed according to the LEFM $K$-field displacement solution, $u_{x}=\frac{K_{1}}{2 G} \sqrt{\frac{r}{2 \pi}}(\kappa-\cos \theta) \cos \frac{\theta}{2}$, $u_{y}=\frac{K_{1}}{2 G} \sqrt{\frac{r}{2 \pi}}(\kappa-\cos \theta) \sin \frac{\theta}{2}, u_{z}=0$, where $G$ is the shear modulus and $\kappa=(3-\eta) /(1+\eta)$. The atoms within a radius of $100 \AA$ of the crack tip were evolved with NVT dynamics at temperature $T=300 \mathrm{~K}$. The radius of $100 \AA$ is chosen because the applied strain beyond this radius is $<0.02$, which is small enough for linear elasticity to be applicable. Also, we note that all bond breaking events occur within a radius of $20 \AA$, thus the simulation box size is large enough to avoid any finite size effects. The applied stress-intensity factor $K_{\mathrm{I}}$ was increased in increments of $0.1 \mathrm{MPa} \cdot \mathrm{m}^{1 / 2}$. The system was held at each value of $K_{\mathrm{I}}$ for $t=1 \mathrm{ps}$. The critical stress-intensity factor was taken to be the lowest value of $K_{\mathrm{I}}$ for which the crack grows. We simulated initial crack advance in polycrystals with grain size $\mu=16,32,64 \AA$. For each grain size, crack advance was simulated in 500 polycrystals with random grain morphology.

\section{References}

1. Castro Neto, A. H., Guinea, F., Peres, N. M., Novoselov, K. S. \& Geim, A. K. The electronic properties of graphene. Rev. Mod. Phys. 81, 109-162 (2009).

2. Novoselov, K. S. et al. Two-dimensional gas of massless Dirac fermions in graphene. Nature 438, 197-200 (2005).

3. Bonaccorso, F., Sun, Z., Hasan, T. \& Ferrari, A. C. Graphene photonics and optoelectronics. Nat. Photon. 4, 611-622 (2010).

4. Balandin, A. A. et al. Superior thermal conductivity of single-layer graphene. Nano Lett. 8, 902-907 (2008).

5. Bae, S. et al. Roll-to-roll production of 30 -inch graphene films for transparent electrodes. Nat. Nanotechnol. 5, 574-578 (2010).

6. Prasai, D., Tuberquia, J. C., Harl, R. R., Jennings, G. K. \& Bolotin, K. I. Graphene: Corrosion-inhibiting coating. ACS Nano 6, 1102-1108 (2012).

7. Liu, Y., Dong, X. \& Chen, P. Biological and chemical sensors based on graphene materials. Chem. Soc. Rev. 41, 2283-2307 (2012).

8. Sun, X. et al. Nano-graphene oxide for cellular imaging and drug delivery. Nano Res. 1, 203-212 (2008).

9. Geim, A. K. \& Novoselov, K. S. The rise of graphene. Nat. Mater. 6, 183-191 (2007).

10. Zhang, P. et al. Fracture toughness of graphene. Nat. Commun. 5, 3782 (2014).

11. Grantab, R., Shenoy, V. B. \& Ruoff, R. S. Anomalous strength characteristics of tilt grain boundaries in graphene. Science 330, 946-948 (2010).

12. Wei, Y. et al. The nature of strength enhancement and weakening by pentagonheptagon defects in graphene. Nat. Mater. 11, 759-763 (2012).

13. Khare, R. et al. Coupled quantum mechanical/molecular mechanical modelling of the fracture of defective carbon nanotubes and graphene sheets. Phys. Rev. B 75, 075412 (2007)

14. Zhang, T., Li, X., Kadkhodaei, S. \& Gao, H. Flaw insensitive fracture in nanocrystalline graphene. Nano Lett. 12, 4605-4610 (2012).

15. Wang, M. C., Yan, C., Ma, L., Hu, N. \& Chen, M. W. Effect of defects on fracture strength of graphene sheets. Comp. Mat. Sci. 54, 236-239 (2012).
16. Rasool, H. I., Ophus, C., Klug, W. S., Zettl, A. \& Gimzewski, J. K. Measurement of the intrinsic strength of crystalline and polycrystalline graphene. Nat. Commun. 4, 2811 (2013).

17. Song, Z., Artyukhov, V. I., Yakobson, B. I. \& Xu, Z. Pseudo Hall-Petch strength reduction in polycrystalline graphene. Nano Lett. 13, 1829-1833 (2013).

18. Ritchie, R. O. The conflicts between strength and toughness. Nat. Mater. 10, 817-822 (2011).

19. Lee, G.-H. et al. High-strength chemical-vapour-deposited graphene and grain boundaries. Science 340, 1073-1076 (2013).

20. Ruiz-Vargas, C. S. et al. Softened elastic response and unzipping in chemical vapour deposition graphene membranes. Nano Lett. 11, 2259-2263 (2011).

21. Suk, J. W., Mancevski, V., Hao, Y., Liechti, K. M. \& Ruoff, R. S. Fracture of polycrystalline graphene membranes by in situ nanoindentation in a scanning electron microscope. Phys. Status Solidi 9, 564-569 (2015).

22. Li, X. et al. Large-area synthesis of high-quality and uniform graphene films on copper foils. Science 324, 1312-1314 (2009).

23. Ren, W. \& Cheng, H.-M. The global growth of graphene. Nat. Nanotechnol. 9 , 726-730 (2014).

24. Hall, E. O. The deformation and ageing of mild steel: III Discussion of results. Proc. Phys. Soc. Sec. B 64, 747-753 (1951).

25. Singh, J. P., Virkar, A. V., Shetty, D. K. \& Gordon, R. S. Strength-grain size relations in polycrystalline ceramics. J. Am. Ceram. Soc. 62, 179-183 (1979).

26. Rice, R. W., Freiman, S. W. \& Mecholsky, J. J. The dependence of strengthcontrolling fracture energy on the flaw-size to grain-size ratio. J. Am. Cer. Soc. 63, 129-136 (1980).

27. Huang, P. Y. et al. Grains and grain boundaries in single-layer graphene atomic patchwork quilts. Nature 469, 389-392 (2011).

28. Yazyev, O. V. \& Louie, S. G. Topological defects in graphene: Dislocations and grain boundaries. Phys. Rev. B 81, 195420 (2010).

29. Banhart, F., Kotakoski, J. \& Krasheninnikov, A. V. Structural defects in graphene. ACS Nano 5, 26-41 (2011).

30. Kim, K. et al. Grain boundary mapping in polycrystalline graphene. ACS Nano 5, 2142-2146 (2011).

31. Kotakoski, J. \& Meyer, J. C. Mechanical properties of polycrystalline graphene based on a realistic atomistic model. Phys. Rev. B 85, 195447 (2012).

32. Liu, T.-H., Pao, C.-W. \& Chang, C.-C. Effects of dislocation densities and distributions on graphene grain boundary failure strengths from atomistic simulations. Carbon 50, 3465-3472 (2012).

33. Sha, Z. D. et al. On the failure load and mechanism of polycrystalline graphene by nanoindentation. Sci. Rep. 4, 7437 (2014).

34. Epstein, B. Statistical aspects of fracture problems. J. Appl. Phys. 19, 140-147 (1948).

35. Weibull, W. A statistical distribution function of wide applicability. J. Appl. Mech. 13, 293-297 (1951).

36. Gulino, R. \& Phoenix, S. L. Weibull strength statistics for graphite fibres measured from the break progression in a model graphite/glass/epoxy microcomposite. J. Mater. Sci. 26, 3107-3118 (1991).

37. Hui, C. Y., Phoenix, S. L. \& Shia, D. The single-filament-composite test: a new statistical theory for estimating the interfacial shear strength and Weibull parameters for fiber strength. Compos. Sci. Technol. 57, 1707-1725 (1998).

38. Tobolsky, A. \& Eyring, H. Mechanical properties of polymeric materials. J. Chem. Phys. 11, 125-134 (1943).

39. Zhurkov, S. N. Kinetic concept of the strength of solids. Int. J. Fract. 26, 295-307 (1965).

40. Phoenix, S. L. \& Tierney, J.-L. A statistical model for the time dependent failure of unidirectional composite materials under local elastic load-sharing among fibers. Eng. Fract. Mech. 18, 193-215 (1983).

41. Zhu, T., Li, J., Samanta, A., Leach, A. \& Gall, K. Temperature and strain-rate dependence of surface dislocation nucleation. Phys. Rev. Lett. 100, 025502 (2008).

42. Zhu, T. \& Li, J. Ultra-strength materials. Prog. Mater. Sci. 55, 710-757 (2010).

43. Sellerio, A. L., Taloni, A. \& Zapperi, S. Fracture size effects in nanoscale materials: the case of graphene. Phys. Rev. Appl. 4, 024011 (2015).

44. Mason, J. K., Lund, A. C. \& Schuh, C. A. Determining the activation energy and volume for the onset of plasticity during nanoindentation. Phys. Rev. B 73, 054102 (2006).

45. Rinaldi, A., P., Friesen, P., C. \& K., S. Sample-size effects in the yield behaviour of nanocrystalline nickel. Acta Mater. 56, 511-517 (2008).

46. Wang, W. et al. Size effects and strength fluctuation in nanoscale plasticity. Acta Mater. 60, 3302-3309 (2012).

47. de Haan, L. Extreme Value Theory and Applications (Springer, 1994).

48. Bouchaud, J.-P. \& Mezard, M. Universality classes for extreme-value statistics. J. Phys. A: Math. Gen. 30, 7997-8015 (1997).

49. Sha, Z. et al. Inverse pseudo Hall-Petch relation in polycrystalline graphene. Sci. Rep. 4, 5991 (2014). 
50. Brenner, D. W. et al. A second-generation reactive empirical bond order (REBO) potential energy expression for hydrocarbons. J. Phys. Condens. Matter 14, 783-802 (2002).

51. Stuart, S. J., Tutein, A. B. \& Harrison, J. A. A reactive potential for hydrocarbons with intermolecular interactions. J. Chem. Phys. 112, 6472-6486 (2000).

52. Jung, G., Qin, Z. \& Buehler, M. J. Molecular mechanics of polycrystalline graphene with enhanced fracture toughness. Extreme Mech. Lett. 2, 52-59 (2015).

53. Lin, T., Evans, A. G. \& Ritchie, R. O. A statistical model of brittle fracture by transgranular cleavage. J. Mech. Phys. Sol. 34, 477-497 (1986).

54. Lin, T., Evans, A. G. \& Ritchie, R. O. Statistical analysis of cleavage fracture ahead of sharp cracks and rounded notches. Acta Metall. 34, 2205-2216 (1986).

55. Ritchie, R. O. Fatigue and fracture of pyrolytic carbon: A damage-tolerant approach to structural integrity and life prediction in 'Ceramic' heart valve prostheses. J. Heart Valve Dis. 5(Suppl 1): 9 (1996).

56. Drory, M. D., Dauskardt, R. H., Kant, A. \& Ritchie, R. O. Fracture of synthetic diamond. J. Appl. Phys. 78, 3083-3088 (1995).

57. Ovid'ko, I. A. \& Sheinerman, A. G. Cracks at disclinated grain boundaries in graphene. J. Phys. D: Appl. Phys. 46, 345305 (2013).

58. Song, Z., Artyukhov, V. I., Wu, J., Yakobson, B. I. \& Xu, Z. Defect-detriment to graphene strength is concealed by local probe: the topological and geometrical effects. ACS Nano 9, 401-408 (2015).

59. Plimpton, S. Fast parallel algorithms for short-range molecular dynamics. J. Comp. Phys. 117, 1-19 (1995).

60. Ophus, C., Shekhawat, A., Rasool, H. I. \& Zettl, A. Large-scale experimental and theoretical study of graphene grain boundary structures. Phys. Rev. B. 92, 20542 (2015).

61. Daivis, P. J. \& Todd, B. A simple, direct derivation and proof of the validity of the SLLOD equations of motion for generalized homogeneous flows. J. Chem. Phys. 124, 194103 (2006).

\section{Acknowledgements}

This work was supported by the Mechanical Behaviour of Materials Program (KC13) at the Lawrence Berkeley National Laboratory, funded by the U.S. Department of Energy, Office of Science, Office of Basic Energy Sciences, Materials Sciences and Engineering Division, under Contract No. DE-AC02-05CH11231. A.S. acknowledges financial support from the Miller Institute for Basic Research in Science, at the University of California, Berkeley, in the form of a Miller Research Fellowship.

\section{Author contributions}

A.S. and R.O.R. conceived the study; A.S. performed the simulations and theoretical calculations; and A.S. and R.O.R. wrote the manuscript.

\section{Additional information}

Supplementary Information accompanies this paper at http://www.nature.com/ naturecommunications

Competing financial interests: The authors declare no competing financial interests.

Reprints and permission information is available online at http://npg.nature.com/ reprintsandpermissions/

How to cite this article: Shekhawat, A. \& Ritchie, R. O. Toughness and strength of nanocrystalline graphene. Nat. Commun. 7:10546 doi: 10.1038/ncomms10546 (2016)

\section{(c) (i)}

This work is licensed under a Creative Commons Attribution 4.0 International License. The images or other third party material in this article are included in the article's Creative Commons license, unless indicated otherwise in the credit line; if the material is not included under the Creative Commons license, users will need to obtain permission from the license holder to reproduce the material. To view a copy of this license, visit http://creativecommons.org/licenses/by/4.0/ 